\title{
Beratung per E-Mail - welche EBM-Ziffer kommt infrage?
}

\begin{abstract}
_ Die Nr. 01100 EBM wird berechnet, wenn ein Patient den Arzt an einem Werktag zwischen 19 und 22 Uhr oder an einem Ruhetag (Samstag, Sonntag, gesetzlicher Feiertag, 24. und 31. Dezember) zwischen 7 und 19 Uhr unvorhergesehen in Anspruch nimmt. Die Nr. 01101 wird berechnet, wenn dies werktags zwischen 22 und 7 Uhr oder an einem Ruhetag zwischen 19 und 7 Uhr geschieht. Auch bei telefonischer Kontaktaufnahme stehen die Nrn. zur Verfügung. Außerhalb dieser Unzeiten käme die „Haus-/Fachärztliche Bereitschaftspauschale" nach Nr. 01435 in Betracht.
\end{abstract}

Doch was ist, wenn der Patient per EMail Kontakt aufnimmt und der Arzt ihm auch sofort antwortet?

\section{MMW-KOMMENTAR}

Die Nr. 01435 kann einmalig im Quartal für eine telefonische Beratung zu einer Krankheit berechnet werden, wenn der Patient den Kontakt aufnimmt - und ist auch bei E-MailKommunikation möglich. Der Nachteil ist, dass dann im ganzen Quartal die Versichertenpauschale ausgeschlossen ist. Kommt der Patient nach einer solchen Kontaktaufnahme also in die Praxis, wird die Leistung wieder gestrichen. Bei Anfragen zur Unzeit ist

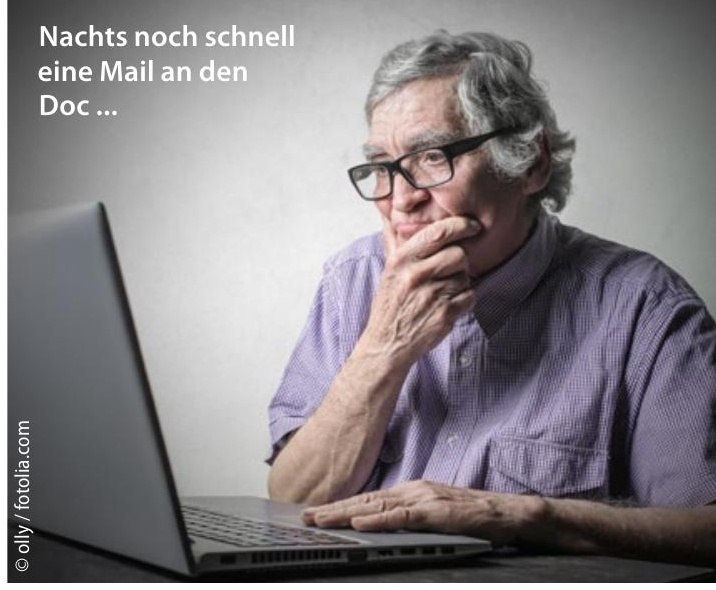

das anders. Diese Nrn. 01100 und 01101 können pro Kontaktaufnahme durch den Patienten berechnet werden und unterliegen noch nicht einmal der Plausibilitätskontrolle nach Zeitvorgaben. Entscheidend ist der Zeitpunkt der Kontaktaufnahme durch den Patienten der Zeitpunkt der Ausführung (Rückmail, Rückruf) spielt keine Rolle.

\section{Transport zur Chemotherapie wird einfacher}

_ Das Bundesministerium für Gesundheit hat die Änderungen der Krankentransport-Richtlinie (MMW 5/2015, S. 31) nicht beanstandet. Der Beschluss des Gemeinsamen Bundesausschusses tritt damit in den kommenden Tagen nach Veröffentlichung im Bundesanzeiger in Kraft. Statt „onkologische Chemothera- pie“ heißt es in der Richtlinie nun also „parenterale antineoplastische Arzneimitteltherapie/parenterale onkologische Chemotherapie“.

\section{MMW-KOMMENTAR}

Konkret bedeutet dies, dass alle Patienten, die einer solchen, nunmehr konkretisierten
Behandlung bedürfen, Transportscheine für einen Behandlungstermin erhalten können, ohne dass eine vorherige Genehmigung der Kasse erforderlich ist. Auch bei Fahrten zur Dialysebehandlung oder zur onkologischen Strahlentherapie braucht man im ambulanten Behandlungsfall keine Genehmigung einzuholen.

\section{Kassendefizit 2015 liegt nicht an den Ärzten!}

- Wie das Statistische Bundesamt mit-
geteilt hat, haben die gesetzlichen Kran-
kenkassen in Deutschland im Jahr 2015
ein finanzielles Defizit von 3,1 Milliar-
den Euro verbucht. Dabei sind die GKV-
Einnahmen einschließlich Gesundheits-
fonds kräftig gestiegen, nämlich um
3,4\% auf 212,3 Milliarden Euro. Die Aus-
gaben stiegen allerdings noch stärker, nämlich um 3,9\% auf nunmehr 215,4 Milliarden Euro.

\section{MMW-KOMMENTAR}

Die Kassen werden die Ursache dieses Defizits wieder einmal bei den sogenannten Leistungserbringern suchen. In Wahrheit liegt es zum einen daran, dass die Krankenkassen vermutlich aus Konkurrenzdruck - von ihren
Versicherten niedrigere Zusatzbeiträge verlangt haben, als es notwendig gewesen wäre. Zum anderen hat die Bundesregierung den Bundeszuschuss um 2,5 auf 11,5 Milliarden Euro abgesenkt. Auch ein eher kleiner Posten von 1,8 Millionen Euro sollte erwähnt werden: Diesen Betrag verlor der Gesundheitsfonds an die Banken, die momentan Strafzinsen für geparktes Geld verlangen. 